\title{
Surgical strategy of brain tumors adjacent to the optic radiation using diffusion tensor imaging-based tractography
}

\author{
KAZUNORI TATSUZAWA ${ }^{1}$, KEI OWADA ${ }^{1}$, HIROYASU SASAJIMA ${ }^{1}$, \\ KEI YAMADA ${ }^{2}$ and KATSUYOSHI MINEURA ${ }^{1}$
}

Departments of ${ }^{1}$ Neurosurgery and ${ }^{2}$ Radiology, Kyoto Prefectural Graduate School of Medicine, Kyoto 602-8566, Japan

Received May 12, 2010; Accepted August 13, 2010

DOI: $10.3892 / \mathrm{ol} .2010 .177$

\begin{abstract}
The present study evaluated the efficacy of diffusion tensor imaging (DTI)-based tractography in the surgery of brain tumors adjacent to the optic radiation. Of the 14 surgical cases included, 11 had metastatic brain tumors and 3 cerebral gliomas. Additionally, 4 of the 14 patients had pre-operative visual field defects, while the remaining 10 patients experienced no visual impairment. The optic radiations on the lesion side were evident in all 14 patients. On the basis of these tractographic findings, we employed optimal surgical approaches in each patient to avoid injury to the eloquent neural structures, including optic radiation, during surgery. Successful surgical resection was performed in all 14 patients. Of the 14 patients, 2 with visual field defects during the pre-operative period showed improvement in their visual field, and the remaining 12 patients experienced no visual deterioration. DTI-based tractography thus is a feasible modality for the surgical planning of brain lesions adjacent to the optic radiation.
\end{abstract}

\section{Introduction}

Intra-axial brain tumors mainly spread within the brain parenchyma causing a variety of motor, sensory, language and visual dysfunctions. Apart from recent advancements in radiochemotherapy, surgery plays an important role in rapidly reducing intracranial pressure and local adverse effects on brain function, resulting in the maintenance of the performance status and prolongation of patient survival.

In the surgery of patients with brain tumors, the principal aim is to preserve both functional cortical gray and white

Correspondence to: Dr Kazunori Tatsuzawa, Department of Neurosurgery, Kyoto Prefectural Graduate School of Medicine, Kawaramachi Hirokoji, Kamigyo-ku, Kyoto 602-8566, Japan E-mail: tatsu@koto.kpu-m.ac.jp

Abbreviations: DTI, diffusion tensor imaging; MRI, magnetic resonance imaging; FLAIR, fluid-attenuated inversion-recovery; fMRI, functional MRI; ROIs, regions of interest; 3D, threedimensional; FA, fractional anisotropy

Key words: brain tumor, diffusion tensor imaging, tractography, optic radiation matter tracts while maximizing surgical resection of the lesions (1-3). In cases of lesions originating in the temporal, parietal, occipital lobe or lateral ventricle, anatomical and functional preservation of the optic radiation is essential (4).

Conventional magnetic resonance imaging (MRI) techniques, such as T2-weighted, gadolinium-enhancing T1-weighted and fluid-attenuated inversion-recovery (FLAIR) imaging, have been widely used for the evaluation of the anatomical location of brain lesions. However, these techniques provide limited information regarding the integrity and location of white matter tracts adjacent to the lesions. Similarly, functional MRI (fMRI) provides limited information on the status of white matter structures, although it localizes eloquent cortical areas surrounding brain lesions $(2,3,5,6)$.

Recent advances in magnetic resonance (MR) technology have enabled major neural tracts to be distinguished in the white matter. Determining the exact location of the lesion with respect to clinically eloquent white matter tracts is significant to neurosurgeons in planning the appropriate surgical approach and in predicting the extent of safe resection (1-4,6-9). The present study evaluated the feasibility of diffusion tensor imaging (DTI)-based tractography in the pre-surgical planning of brain tumors adjacent to the optic radiation.

\section{Materials and methods}

Patient population. This study was approved by the Internal Review Board of our institution, and written informed consent for the MR studies was obtained from each patient. Between May 2004 and February 2009, DTI was performed on 14 consecutive patients with brain tumors adjacent to the optic radiation. Patients ranged in age from 27 to 75 years (mean 55). Lesions consisted of metastatic tumors in 11 patients and glioma in 3. The location of lesions was the occipital lobe in 7 patients, the temporal lobe in 5 and the parietal lobe in 2 patients.

MRI techniques. MR images were obtained with a whole-body 1.5-T MR system (Gyroscan Intera; Philips Medical Systems, Best, The Netherlands) with a gradient strength of $30 \mathrm{mT} / \mathrm{m}$. A single-shot echo-planar technique was used to obtain the diffusion-weighted MR images (repetition time/echo time, 6,000/58 msec) with a motion-probing gradient in 32 orientations and a field of view of $230 \mathrm{~mm}$. B-values of 0 and $1,000 \mathrm{sec} / \mathrm{mm}^{2}$ were used with averages of three images. The $128 \times 58$ data 
Table I. Characteristics and outcome of the 14 patients examined with diffusion tensor imaging.

\begin{tabular}{|c|c|c|c|c|c|c|c|c|}
\hline No. & Gender & Age & Diagnosis & Location & Size $(\mathrm{cm})$ & Visual disturbance & Removal & Neurological outcome \\
\hline 1 & $\mathrm{~F}$ & 63 & Metastasis & Occipital lobe & 2 & None & Total & $\mathrm{NC}$ \\
\hline 2 & $\mathrm{~F}$ & 60 & Metastasis & Occipital lobe & 3 & Quad & Total & $\mathrm{NC}$ \\
\hline 3 & $\mathrm{~F}$ & 46 & Metastasis & Occipital lobe & 3 & None & Total & $\mathrm{NC}$ \\
\hline 4 & M & 75 & Metastasis & Occipital lobe & 3 & None & Partial & $\mathrm{NC}$ \\
\hline 5 & $\mathrm{~F}$ & 55 & Metastasis & Occipital lobe & 4 & None & Total & $\mathrm{NC}$ \\
\hline 6 & M & 63 & Metastasis & Occipital lobe & 4 & None & Partial & $\mathrm{NC}$ \\
\hline 7 & M & 42 & Metastasis & Occipital lobe & 5 & Hemi & Total & Improved \\
\hline 8 & $\mathrm{~F}$ & 60 & Metastasis & Parietal lobe & 3 & None & Total & $\mathrm{NC}$ \\
\hline 9 & M & 74 & Metastasis & Parietal lobe & 5 & None & Total & $\mathrm{NC}$ \\
\hline 10 & $\mathrm{~F}$ & 55 & Metastasis & Temporal lobe & 5 & None & Total & $\mathrm{NC}$ \\
\hline 11 & M & 41 & Metastasis & Temporal lobe & 7 & Hemi & Total & Improved \\
\hline 12 & $\mathrm{~F}$ & 27 & Glioma & Temporal lobe & 2 & None & Subtotal & $\mathrm{NC}$ \\
\hline 13 & M & 42 & Glioma & Temporal lobe & 3 & Quad & Subtotal & $\mathrm{NC}$ \\
\hline 14 & M & 65 & Glioma & Temporal lobe & 4 & None & Subtotal & $\mathrm{NC}$ \\
\hline
\end{tabular}

Quad, quadrantanopsia; Hemi, hemianopsia; NC, no change.

points were recorded by using the parallel imaging technique. The parallel-imaging technique allows image reconstruction with half encoding steps, the advantage of which lies in its reduction in the geometric image distortion that is unique to echo-planar imaging. A total of 36 slices with a thickness of $2 \mathrm{~mm}$ and without interslice gaps were obtained $(5,10)$. The DTI studies were performed at the end of the routine work-up for the pre-surgical evaluation of brain tumors at our institute with an average acquisition time of $7 \mathrm{~min}$.

Data processing. The DTI data were transferred to an offline workstation and then PRIDE software was used (Philips Medical Systems) for image analysis. Diffusion tensor elements and anisotropy at each voxel were calculated, followed by the construction of color maps based on the DTI from these data for the vector in the longest axis (v1). Vector elements were allocated different colors: red represented the $x$ element (left to right), green the $y$ element (anteroposterior) and blue the $\mathrm{z}$ element (superoinferior). The intensity of the maps was scaled in proportion to the fractional anisotropy (FA) $(5,10)$.

Fiber tracking. The procedure for mapping neural connections commenced by designating two arbitrary regions of interest (ROIs) in the three-dimensional (3D) space. To visualize the optic radiation, a pair of ROIs was placed on the lateral geniculate nucleus of the thalamus and near the primary visual cortex, including the calcarine fissure in the two hemispheres on the coronal vector-color map. The extent of the axonal projections was traced from the seed pixels within the ROIs in the anterograde (forward) and retrograde (backward) directions. Tracking was terminated (stop criterion) when a pixel with low FA, a pre-determined trajectory curvature between two contiguous vectors, or both were reached. In cases when no continuous tracts between two previously defined ROIs were confirmed, one ROI at the lateral geniculate nucleus and an additional large ROI covering a wide area of the ipsilateral occipital lobe were employed. Consequently, fiber tracts passing through the two ROIs were identified as the final tract of interest. The FA and/or a pre-determined trajectory curvature between two contiguous vectors (inner product) were variously defined until final values that provided the most complete reconstruction of the tracts of interest were determined $(5,10)$.

Assessment and treatment. On the basis of the pre-surgical fiber tracking results overlaid in the T2-weighted MR image, the structural integrity and location of the eloquent optic radiation relevant to the brain tumors were determined. Conventional MR images, such as T1-, and T2-weighted MR imaging, FLAIR MR imaging, and gadolinium-enhancing T1-weighted MR imaging in three orthogonal directions were evaluated. Moreover, surgically significant extracranial and intracranial vascular structures were thoroughly studied by means of MR angiography, 3D computerized tomography angiography or conventional cerebral angiography when necessary. Finally, optimal surgical approaches were employed in each patient to avoid injury to the eloquent cortex and white matter as well as to preserve normal vascular structures to the utmost extent.

Pre-operative and postsurgical visual field defects were assessed by ophthalmologists using the Goldmann perimeter.

\section{Results}

The optic radiations on the lesional side were evident in all 14 patients, and the exact location of each brain tumor relevant to the eloquent white matter tracts was discernible. In all 14 cases, the optic radiation was displaced superiorly, inferiorly or medially, depending on the location of the lesion, and no evidence of white matter infiltration by each brain tumor was noted.

A total of 11 metastatic tumors and 3 gliomas (2 anaplastic astrocytomas and 1 glioblastoma) were extensively resected using the transcortical approach. Thus, violation to the eloquent 

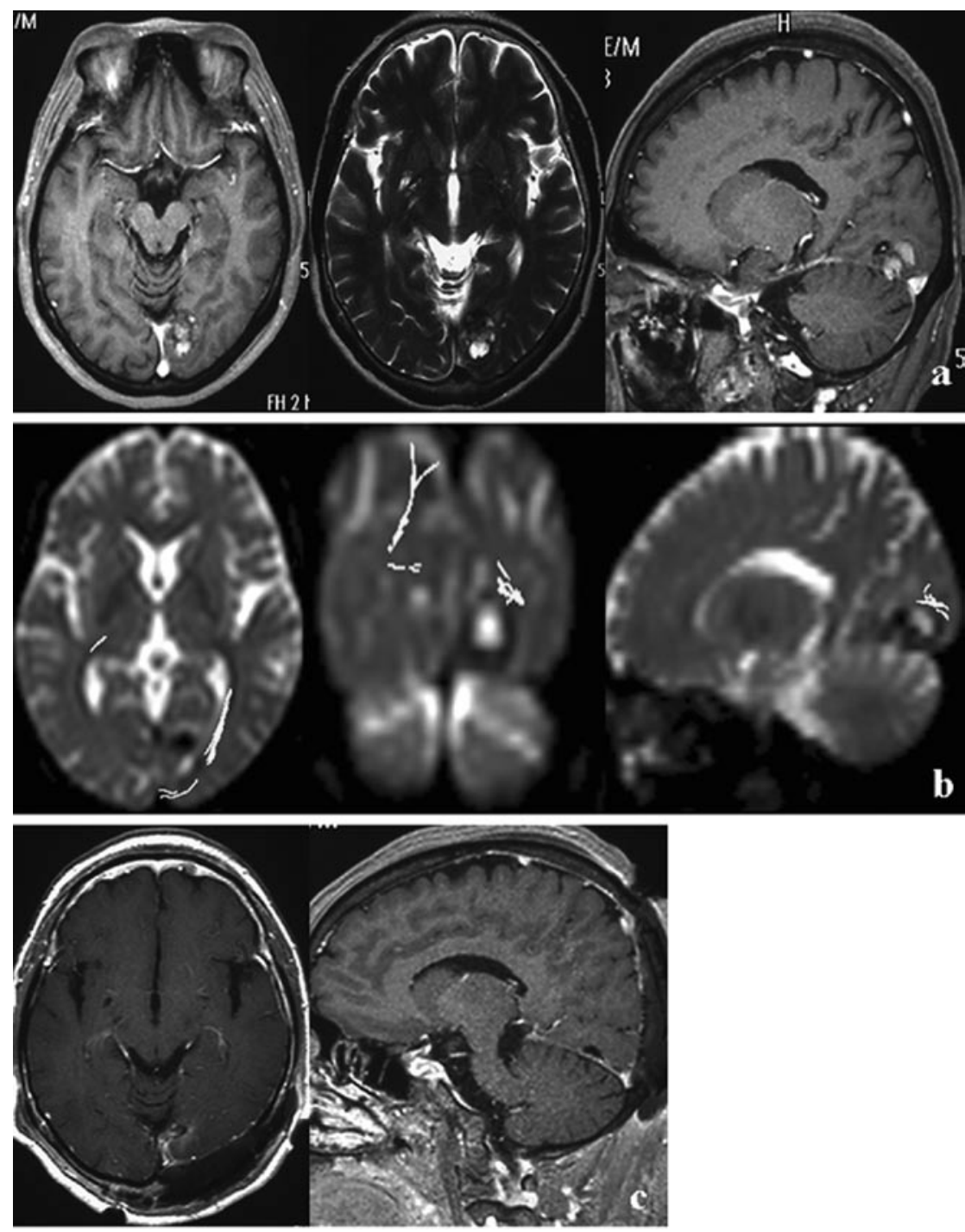

Figure 1. Neuroimaging of an illustrative case. (a) Pre-operative T2 and gadolinium-enhancing MRI shows a heterogeneously enhanced mass of the visual cortex. (b) Pre-operative tractography shows a superolateral shifting of the optic radiation on the lesional side. (c) Postoperative MRI illustrates total extirpation of the tumor.

cortex was avoided to the utmost extent, while the shortest surgical tract to each brain lesion was selected. Debulking the tumor and obtaining sufficient working space was initially carried out. Maximal delicate surgical manipulation, including the limitation of both mechanical and heat injury, was then achieved particularly for the lesion adjacent to the supposed optic radiation.

One of the 2 patients presenting with quadrantanopsia and 1 of the 2 patients with hemianopsia during the pre-operative period showed improvement in their visual field postoperatively. The remaining 2 patients with pre-operative visual defects and 10 patients with normal visual function experienced no visual deterioration after surgery. Table I shows the characteristics and surgical results of the patients.

Illustrative case. A 63-year-old woman with a medical history of lung cancer presented to our institute after experiencing a sudden onset of headaches. An examination showed no evidence of neurological deficits, and a Goldmann perimeter showed a full visual field. Conventional MRI revealed a $2-\mathrm{cm}$ heterogeneous signal mass lesion on the T1- and T2-weighted images in the left occipital lobe. The lesion was accompanied with repeated hemorrhage during the follow-up period (Fig. 1a). Pre-operative diffusion-tensor tractography showed that the optic radiation was deviated superiorly and medially relative to the mass lesion (Fig. 1b). The patient underwent a left occipital craniotomy, and gross total resection of the mass lesion was performed using the transcortical lingual gyrus approach (Figs. 1c and 2a and b). The lesion was intentionally approached through an inferior occipital surface for preserving the eloquent optic radiation running at the opposite end. Pathological findings were consistent with a metastatic adenocarcinoma. The patient was discharged from our institute without any neurological deficit.

\section{Discussion}

In planning the surgery of brain lesions adjacent to the optic radiation, it is important to pre-operatively confirm the $3 \mathrm{D}$ relationship between the lesion and affected tract as well 


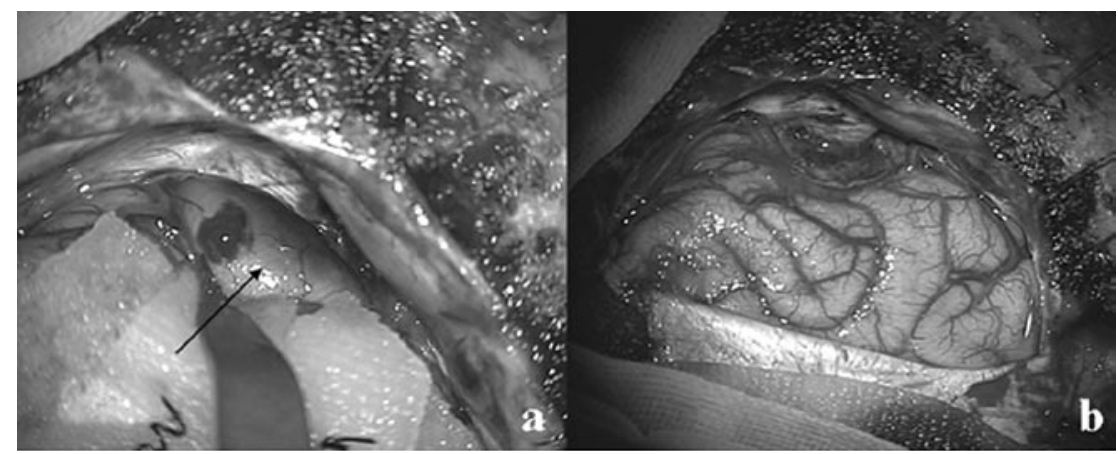

Figure 2. Intra-operative images of the illustrative case. (a) The arrow indicates the darkly discolored cortex, including perilesional hemorrhage. (b) Gross total resection was performed on the tumor using the inferior occipital approach.

as the eloquent gray matter, including the visual cortex, to avoid postoperative permanent visual impairments $(1,4,7)$. The usefulness of functional mapping using fMRI, positronemission tomography or visual evoked potential in surgical planning was previously confirmed in patients with occipital lobe tumors (11-13).

DTI provides information on the direction water molecules follow at the cellular level, indicating the orientation of fiber tracts. DTI-based tractography is currently the only imaging method used to show white matter tracts in vivo $(5,14)$. This technique is widely used to visualize motor, sensory, speech and visual pathways for neurosurgical planning and postoperative assessment $(1,3)$. Our imaging technique facilitated the visualization of targeted white matter in a short time period and with less invasiveness. This technique is also feasible for patients with a poor performance status.

The optic radiation starts in the lateral geniculate body and forms a band that winds around the inferior and posterior horns of the lateral ventricles, terminating in the visual cortex or striate area, with the three-bundled arrangement comprising the anterior, central and posterior bundles $(10,15)$. The anterior bundle runs anteriorly prior to making a sharp turn around the tip of the inferior horn (known as Meyer's loop) and passes posteriorly along the lateral wall of the ventricle ultimately terminating on the lower lip of the calcarine fissure (16). The central bundle initially runs in the lateral direction, then posteriorly along the lateral wall of the ventricle and finally radiates into the calcarine cortex. In addition, the posterior bundle runs directly in the posterior direction along the roof of the ventricle and converges on the upper lip of the calcarine fissure. Specifically, the anterior bundle subserves the superior quadrant; the central bundle, the macula; and the posterior bundle, the inferior visual quadrant $(10,15)$. Particularly in the pre-surgical planning of anterior temporal lobe resection in patients with refractory temporal lobe epilepsy due to hippocampal sclerosis and resection of tumors originating in medial temporal structures or within the temporal horn, knowledge of the boundary of the anterior fibers of the Meyer's loop and their relationship to the temporal pole is required, but remains problematic $(15,16)$. A recent study demonstrated that tractography of the optic radiation is clinically feasible in patients undergoing temporal lobe resections in spite of the technical difficulty of visualizing the anterior extent of Meyer's loop (17).
Prior to the surgical exposure of tumors seated in the temporal, occipital or parietal lobe, our neuroimaging technique assessed the anatomical relationships between the optic radiations and these lesions. The aim of surgery for brain tumors adjacent to the functional cortical gray and white matter tracts is to preserve vital cerebral tissue in order to avoid postoperative neurological deficits as well as to maximize lesion resectability (1-3). In patients with mass lesions located in the posterior part of the temporal lobe or in the temporo-parietooccipital junction, we selected an appropriate surgical tract that was distant to the eloquent fiber running beside the lesion. Subsequently, less surgical manipulation of the visual tracts occurred and violation of the functionally eloquent cortex, areas involved in vision or language, during cortical incision was avoided. On the other hand, we used the subtemporal approach through the fusiform or parahippocampal gyri or the inferior temporal gyrus approach particularly to avoid Meyer's loop, resulting in the safe resection of the medial temporal tumors (15).

In the present study, the efficacy of tractography was tested to determine the appropriate surgical corridor and the extent of resection in patients with brain tumors adjacent to the optic radiations. Tractography successfully designated the site of cortical incision in combination with the findings of conventional MRI, MR angiography, MR venography and conventional angiography. It was previously reported that the combination of fMRI and DTI-based tractography may permit the achievement of more favorable results in pre-surgical planning in brain tumor resection $(2,8,14)$. In all 14 patients, tractography facilitated the visualization of the geniculocalcarine tract passing close to the lesions. The size and site of lesions, as well as perifocal changes such as edema and hemorrhage, may interfere with the visualization of the optic tracts $(5,6)$. In the present study, the optic tracts were examined for large lesions (up to $7 \mathrm{~cm}$ ) with or without perifocal edema in the pre-surgical planning stage. Regarding the site, the relationship of the posterior temporal, occipital or parietal lesions to the optic radiation posterior to the geniculate ganglion was achieved, and the choice of surgical approach was facilitated. In contrast, in a few cases of medial temporal lesions, technical difficulty in obtaining the trajectory curvature of Meyer's loop on DTI occurred. Consequently, the interpretation of tractographic images should be undertaken with caution due to considerable heterogeneity in the anterior extent (17). 
To predict the anatomical relationship between the eloquent fiber and the mass lesion, an understanding of the pathological behavior of the lesions is crucial, i.e., whether they are expansive or infiltrative. Observation with an operative microscope clearly shows the demarcated border in the majority of expansive growth tumors, such as metastatic ones. Therefore, peritumoral structure, including the white matter bundle, may tend to be displaced by a mass effect. During the resection of expansive growth tumors, it is essential to avoid violation of the displaced optic radiation through mechanical or heat injury. After achieving a sufficient surgical working space by maximal internal decompression, tumor dissection near the presumed eloquent fiber should be meticulous, and the vascular component feeding the eloquent neural tissue should be similarly preserved. Consequenlty, gross total removal can be achieved without permanent neurological deficit in the majority of cases.

In contrast, infiltrative growth tumors such as glioma may easily invade or involve the contiguous eloquent fiber. Unlike well-demarcated lesions, surgery of infiltrative lesions is more likely to cause deficits. Therefore, intra-operatively, while a maximal resection of a poorly demarcated mass is attempted, the dissection of white matter bundles around the optic radiations noted in the pre-operative DTI sequences is limited to avoid postoperative neurological deterioration. Reports have described the need for pre-operative and intraoperative integrated neurosurgical planning combined with electrophysiological and functional image guidance to define the safe resection limit $(1,8,9,11,13)$. Multimodal navigation, in addition to pre-operative tractography, in the microsurgical management of eloquent lesions is expected to allow surgeons to maximize surgical resectability, as well as to minimize postoperative morbidity and prolong patient survival.

At the time of surgical planning, the reliability of tractographic images in defining trajectories that are anatomically compatible with eloquent fiber should be considered (14). An important limitation to the clinical use of DTI-based tractography is poor quantification. The threshold of degrees, whether there is clinical significance or not in tract visualization, remains to be determined. It is reported that intra-operative subcortical neurostimulation may underestimate the fiber tracts (18). However, other authors report the accordance of findings of DTI tractography and subcortical stimulation $(4,11)$.

Using this innovative technique, we showed the anatomical relationship between brain tumors and the optic radiations. However, tractography is a subjective process, dependent on user-defined parameters, and the visualized tract themselves are 'virtual' representations of the underlying white matter (17). Therefore, further studies are required to validate this method and establish clinical significance through pre-operative imaging, intra-operative electrophysiological data and postoperative clinico-radiological results $(3,13)$.

In conclusion, our study showed that DTI tractography is clinically feasible and provides useful information regarding the surgical strategy for lesions located in eloquent visual areas. Despite the existence of limitations, improvements in pre-operative visual dysfunction and the absence of postoperative deficits indicate the potential of this technique as a neurosurgical planning tool.

\section{References}

1. Romano A, D'Andrea G, Minniti G, Mastronardi L, Ferrante L, Fantozzi LM and Bozzao A: Pre-surgical planning and MR-tractography utility in brain tumour resection. Eur Radiol 19: 2798-2808, 2009.

2. Witwer BP, Moftakhar R, Hasan KM, et al: Diffusion-tensor imaging of white matter tracts in patients with cerebral neoplasm. J Neurosurg 97: 568-575, 2002.

3. Yu CS, Li KC, Xuan Y, Ji XM and Qin W: Diffusion tensor tractography in patients with cerebral tumors: a helpful technique for neurosurgical planning and postoperative assessment. Eur J Radiol 56: 197-204, 2005.

4. Shinoura N, Suzuki Y, Yamada R, Tabei Y, Saito K and Yagi K: Relationships between brain tumor and optic tract or calcarine fissure are involved in visual deficits after surgery for brain tumor. Acta Neurochir 152: 637-642, 2010.

5. Yamada K, Kizu O, Mori S, et al: Brain fiber tracking with clinically feasible diffusion-tensor MR imaging: initial experience. Radiology 227: 295-301, 2003.

6. Clark CA, Barrick TR, Murphy MM and Bell BA: White matter fiber tracking in patients with space-occupying lesions of the brain: a new technique for neurosurgical planning? Neuroimage 20: 1601-1608, 2003.

7. Kikuta K, Takagi Y, Nozaki K, et al: Early experience with 3-T magnetic resonance tractography in the surgery of cerebral arteriovenous malformations in and around the visual pathway. Neurosurgery 58: 331-337, 2006.

8. González-Darder JM, González-López P, Talamantes F, Quilis V, Cortes V, Garcia-March G and Rordán P: Multimodal navigation in the functional microsurgical resection of intrinsic brain tumors located in eloquent motor areas: role of tractography. Neurosurg Focus 28: E5, 2010.

9. Nimsky C, Ganslandt O, Hastreiter P, Wang R, Benner T, Sorensen AG and Fahlbusch R: Preoperative and intraoperative diffusion tensor imaging-based fiber tracking in glioma surgery. Neurosurgery 56: 130-138, 2005.

10. Yamamoto $T$, Yamada $K$, Nishimura $T$ and Kinoshita $S$ : Tractography to depict three layers of visual field trajectories to the calcarine gyri. Am J Ophthalmol 140: 781-785, 2005.

11. Kamada K, Todo T, Morita A, et al: Functional monitoring for visual pathway using real-time visual evoked potentials and optic-radiation tractography. Neurosurgery 57: 121-127, 2005.

12. Roux FE, Ibarrola D, Lotterie JA, Chollet F and Berry I: Perimetric visual field and functional MRI correlation: implications for image-guided surgery in occipital brain tumours. J Neurol Neurosurg Psychiatry 71: 505-514, 2001.

13. Duffau H, Velut S, Mitchell MC, Gatignol P and Capelle L: Intra-operative mapping of the subcortical visual pathways using direct electrical stimulations. Acta Neurochir 146: 265-270, 2004.

14. Yamada K, Sakai K, Akazawa K, Yuen S and Nishimura T: MR tractography: a review of its clinical applications. Magn Reson Med Sci 8: 165-174, 2009.

15. Sincoff EH, Tan Y and Abdulrauf SI: White matter fiber dissection of the optic radiations of the temporal lobe and implications for surgical approaches to the temporal horn. J Neurosurg 101: 739-746, 2004.

16. Powell HWR, Parker GJM, Alexander DC, et al: MR tractography predicts visual field defects following temporal lobe resection. Neurology 65: 596-599, 2005.

17. Yogarajah M, Focke NK, Bonelli S, et al: Defining Meyer's loop-temporal lobe resections, visual field deficits and diffusion tensor tractography. Brain 132: 1656-1668, 2009.

18. Kinoshita M, Yamada K, Hashimoto N, et al: Fiber-tracking does not accurately estimate size of fiber bundle in pathological condition: initial neurosurgical experience using neuronavigation and subcortical white matter stimulation. Neuroimage 25: 424-429, 2005. 